\title{
Alterações dos parâmetros clínicos e laboratoriais em pacientes obesos com diabetes melito tipo 2 submetidos à derivação gastrojejunal em y de Roux sem anel
}

\section{Changes in clinical and laboratory parameters in obese patients with type 2 diabetes mellitus submitted to Roux-en-y gastrojejunal bypass without ring}

Michelle Varaschim '; Paulo Afonso Nunes Nassif, TCBC-PR²; Luciana Bugmann Moreira3; Marcelo Mazza do Nascimento 3 ; Grasiela Manfredi Nunes Vieira ${ }^{4}$; Rodrigo Ferreira Garcia ${ }^{5}$; Karen Megumi Sue ${ }^{6}$; Manuela Aguiar Cruz ${ }^{6}$

R E S U M O

\begin{abstract}
Objetivos: Verificar as alterações do peso e índice de massa corporal em pacientes obesos grau II e III com diabete melito tipo 2 nos períodos pré e pós-operatório e as alterações dos parâmetros laboratoriais de glicemia de jejum, glicemia pós-prandial, hemoglobina glicada, insulina nos períodos pré e pós-operatório. Métodos: Realizou-se estudo prospectivo selecionando 40 pacientes com obesidade grau II e III, submetidos à derivação gastrojejunal em Y-de-Roux sem anel. Analisou-se no pré e pós-operatório de 60 dias o peso, índice de massa corporal, glicemia de jejum, glicemia pós-prandial, hemoglobina glicada e insulina. Resultados: O peso médio pré-operatório foi de $107,3 \mathrm{Kg}$ diminuindo para $89,5 \mathrm{Kg}$ no pós-operatório. O índice de massa corporal médio inicial foi de $39,5 \mathrm{Kg} / \mathrm{m} 2$ e $32,9 \mathrm{Kg} / \mathrm{m} 2 \mathrm{com} 60$ dias de pós-operatório. A glicemia de jejum no pré-operatório foi de $132 \mathrm{mg} / \mathrm{dl}$ e no pós-operatório diminuiu 40,4 mg/dl em média. A glicemia pós-prandial foi de 172 mg/dl no pré-operatório e 111,6 mg/dl no controle pós-operatório. A hemoglobina glicada inicial foi de $7 \%$ declinando para 5,7\% no pós-operatório. A insulina pré-operatória foi 29,6 ulU/ml e a pósoperatória 13,9 ulU/ml. Todas as variáveis apresentaram significância estatística com $p<0,001$. Conclusão: Houve significante diminuição de peso e no índice de massa corporal entre os períodos pré e pós-operatórios e diminuição também significante dos parâmetros laboratoriais de glicemia de jejum, glicemia pós-prandial, hemoglobina glicada, insulina entre os mesmos períodos.
\end{abstract}

Descritores: Pacientes. Obesidade. Diabetes mellitus tipo 2. Derivação gástrica. Cirurgia Bariátrica.

INTRODUÇÃO

$\mathrm{O}$ acúmulo de tecido gorduroso causado por doenças genéticas ou endócrino-metabólicas ou por alterações nutricionais é denominado obesidade".

A associação entre obesidade e diabetes melito tipo 2 (DM2) é bem definida. A gordura, principalmente a localizada na região abdominal, pode elevar o risco de desenvolver DM2 em 10 vezes. Para cada aumento de $10 \%$ no peso corporal, há aumento de $2 \mathrm{mg} / \mathrm{dL}$ na glicemia em jejum². Cerca de $90 \%$ dos portadores de DM2 estão acima do peso ou são considerados obesos ${ }^{3}$.

A prevalência do DM2 tem tido crescimento em grandes proporções. Estima-se que aproximadamente 150 milhões de pessoas no mundo sofram de DM2, com aumento para aproximadamente 300 milhões em $2025^{4}$.
As bases para o tratamento do diabete melito consistem na manutenção dos valores glicêmicos e de insulinas normais. Esse controle é considerado a melhor abordagem para prevenir complicações agudas e crônicas da doença. Os valores glicêmicos adequados não são atingidos na maioria dos pacientes diabéticos com o tratamento medicamentoso, por eles apresentarem-se acima do peso e resistentes ao controle dietético ${ }^{5}$.

As respostas insatisfatórias com o tratamento medicamentoso levaram ao desenvolvimento de novas modalidades terapêuticas aumentando o interesse na cirurgia bariátrica, que apresenta remissão da doença através da provável recuperação e conservação da função das células beta do pâncreas ${ }^{6}$.

Apesar de ter como objetivo inicial a perda de peso, foi observada importante melhora ou até mesmo o

\footnotetext{
Trabalho realizado no Programa de Pós-Graduação em Princípios da Cirurgia da Faculdade Evangélica do Paraná/Hospital Universitário Evangélico de Curitiba e no Centro Médico do Hospital Universitário Evangélico de Curitiba, Curitiba, PR, Brasil

1. Mestre do Programa de Pós Graduação em Princípios da Cirurgia do Instituto de Pesquisas Médicas da Faculdade Evangélica do Paraná/ Hospital Universitário Evangélico de Curitiba, Curitiba, PR, Brasil; 2. Professor permanente do Programa de Pós Graduação em Princípios da Cirurgia do Instituto de Pesquisas Médicas da Faculdade Evangélica do Paraná; Coordenador do Serviço de Cirurgia Bariátrica e Metabólica do Hospital Universitário Evangélico de Curitiba, Curitiba, PR, Brasil; 3. Professor permanente do Programa de Pós Graduação em Princípios da Cirurgia do Instituto de Pesquisas Médicas da Faculdade Evangélica do Paraná/Hospital Universitário Evangélico de Curitiba, Curitiba, PR, Brasil; 4. Aluno do Programa de Pós Graduação em Princípios da Cirurgia do Instituto de Pesquisas Médicas da Faculdade Evangélica do Paraná/Hospital Universitário Evangélico de Curitiba, Curitiba, PR, Brasil; 5. Cirurgião do Serviço de Cirurgia Bariátrica e Metabólica do Hospital Universitário Evangélico de Curitiba, Curitiba, PR, Brasil; 6. Aluno de Iniciação Científica da Faculdade Evangélica do Paraná/Hospital Universitário Evangélico de Curitiba, Curitiba, PR, Brasil.
} 
controle definitivo do DM2 dos pacientes após a derivação gastrojejunal em Y-de-Roux; da mesma forma, Pories et al. ${ }^{7}$ relataram remissão da doença em $82,9 \%$ dos pacientes após este procedimento cirúrgico. Além desta técnica operatória há relatos de outros tipos de operações bariátricas resultando em melhora clinica do DM2, após a perda de peso

Os mecanismos responsáveis pela perda de peso e pelo controle das doenças associadas incluem não apenas o efeito restritivo e disabsortivo, mas também efeitos hormonais responsáveis pelo controle do apetite e velocidade do trânsito intestinal do alimento9 .

A derivação gastrojejunal em Y-de-Roux é vista como uma operação sacietógena-incretínica devido aos mecanismos hormonais envolvidos na perda de peso e na meIhora das comorbidades, principalmente o DM2. Existe intensa redução na ingestão alimentar acompanhada de paradoxal redução do apetite, atribuída à redução do hormônio grelina na exclusão do fundo gástrico, caracterizando o efeito sacietógeno desse procedimento ${ }^{10}$. A reversão do DM2 envolve também a recuperação da primeira fase da secreção da insulina, que decorre do aumento do glucagon like peptide 1 (GLP-1) com ação incretínica, a qual está aumentada na derivação jejunoileal ${ }^{11,12}$. A operação derivação gastrojejunal em Y-de-Roux é considerada padrão-ouro para o paciente obeso mórbido portador de $\mathrm{DM} 2^{13}$.

Este trabalho tem como objetivo verificar as alterações do peso e índice de massa corporal (IMC) em pacientes com obesidade grau II e III portadores de diabete melito tipo 2 nos períodos pré e pós-operatório, e as alterações dos parâmetros laboratoriais de glicemia de jejum, glicemia pós-prandial, hemoglobina glicada e insulina nas mesmas condições.

\section{MÉTODOS}

O presente estudo foi realizado no Serviço de Cirurgia Bariátrica e Metabólica e no Centro Médico do Hospital Universitário Evangélico de Curitiba. Foi aprovado pelo Comitê de Ética em Pesquisa da Sociedade Evangélica Beneficente.

A amostra foi prospectiva composta por $40 \mathrm{pa}-$ cientes com idade variando de 18 e 65 anos de ambos os sexos (média de 41 anos e com obesidade grau II e III (IMC acima $35 \mathrm{~kg} / \mathrm{m}^{2}$ ). A variação do peso deveria ter sido menor que $10 \%$ nos últimos três meses. Todos pacientes tinham indicação cirúrgica para tratamento da obesidade e eram portadores de diabete melito tipo $2 \mathrm{com}$ menos de 10 anos de evolução. Deveriam estar em uso de hipoglicemiante oral e/ou insulinoterapia (em caso de uso de insulina o período não deveria exceder cinco anos), com nível sérico de peptídeo C maior do que $1 \mathrm{ng} / \mathrm{mL}$. Foram excluídos pacientes com história de doença hepática como cirrose ou hepatite crônica ativa; com alteração da função renal (creatinina maior 1,4 mg/dl em muIheres e maior que 1,5 mg/dl em homens); hepática (alanima aminotransferase e/ou aspartato aminotransferase três vezes acima do valor normal); grávidas; história recente de neoplasia (menos que cinco anos); utilizando corticóide via oral ou injetável por mais de 14 dias consecutivos nos últimos três meses; com sinal laboratorial de provável falência de produção de insulina (peptídeo $\mathrm{C}$ menor que $1 \mathrm{ng} / \mathrm{mL}$ ); presença de anticorpo anti-GAD e/ou anti-insulina e/ou anti-ilhota positivo(s); alcoolismo; drogadição; histórico de doença auto-imune; HIV positivo; e portadores de doenças psiquiátricas descompensadas ou não passíveis de controle.

Dos pacientes selecionados para o estudo foi coletado material pré-operatório para os seguintes exames: glicemia de jejum, hemoglobina glicada, glicemia pósprandial, insulina. Também foram aferidos os valores do peso e IMC.

Com 60 dias de pós-operatório foram realizados os mesmos exames laboratoriais e aferidos os valores de peso e IMC.

O procedimento operatório consistiu em acesso por laparoscopia da cavidade peritoneal, com secção do estômago por grampeamento linear de forma a delimitar um reservatório gástrico junto à cárdia, com capacidade de aproximadamente $20 \mathrm{ml}$, constituindo o estômago funcional pós-operatório. O restante do estômago, assim como o duodeno e os primeiros $80 \mathrm{~cm}$ de jejuno ficavam permanentemente excluídos do trânsito alimentar. O reservatório gástrico era anastomosado à uma alça jejunal isolada em Y- de-Roux e seu esvaziamento limitado por um orifício de 1,3 cm de diâmetro. As secreções provenientes do estômago e do duodeno exclusos desembocam no jejuno por uma anastomose a $100 \mathrm{~cm}$ do ângulo duodenojejunal ${ }^{14}$ (Figura 1).

Para avaliação do efeito da operação sobre as variáveis quantitativas foi considerado o teste t de Student para amostras pareadas. A condição de normalidade das variáveis foi avaliada pelo teste de Kolmogorov-Smirnov. Valores de $p<0,05$ indicaram significância estatística. Os dados foram analisados com o programa computacional Statistica v.8.0.

\section{RESULTADOS}

\section{Variável peso}

Foi obtida diminuição estatisticamente significativa no período analisado $(p<0.001)$. Observou-se que a perda média de peso foi de $17,8 \mathrm{Kg}$ entre o período préoperatório e 60 dias de pós-operatório. O peso médio préoperatório encontrado foi de 107,3 kg.

\section{Variável IMC}

Entre os períodos analisados o IMC apresentou diminuição estatisticamente significativa $(p<0.001)$. Observou-se que entre o período pré-operatório e 60 dias de pós-operatório diminuição média de $6,6 \mathrm{Kg} / \mathrm{m}^{2}$. O IMC pré- 
operatório médio encontrado foi de $39,5 \mathrm{Kg} / \mathrm{m} 2$ enquanto o do pós-operatório médio foi de $32,9 \mathrm{Kg} / \mathrm{m}^{2}$.

\section{Variável glicemia de jejum}

O valor médio da glicemia de jejum no período pré-operatório foi $132 \mathrm{mg} / \mathrm{dl}$ e o no pós-operatório com 60 dias foi $91,6 \mathrm{mg} / \mathrm{dl}$. Observou-se que entre o período préoperatório e 60 dias de pós-operatório a queda média da glicemia de jejum foi de 40,4 mg/dl $\mu$. Foi obtida diminuição estatisticamente significativa da glicemia de jejum entre os períodos analisados ( $p<0.001$ ) (Figura 2).

\section{Variável glicemia pós-prandial}

Os resultados da análise dos valores de glicemia pós-prandial indicaram que existiu diferença significativa entre os valores do período pré-operatório e do período pós-operatório com 60 dias $(p<0,001)$. A glicemia pósprandial pré-operatória média foi de $172 \mathrm{mg} / \mathrm{dl}$ declinando para 111,6 mg/dl após 60 dias do procedimento operatório. A diminuição média da glicemia pós-prandial foi de $60,5 \mathrm{mg} / \mathrm{dl}$ (Figura 3).

\section{Variável hemoglobina glicada}

$\mathrm{Na}$ análise desta variável verificou-se que entre o período pré e pós-operatório houve uma diminuição da hemoglobina glicada com diferença estatisticamente significativa $(p<0,001)$. A média da hemoglogina glicada préoperatória foi de $7 \%$ e da pós-operatória com 60 dias de $5,7 \%$, constatando queda média de 1,3\% (Figura 4).

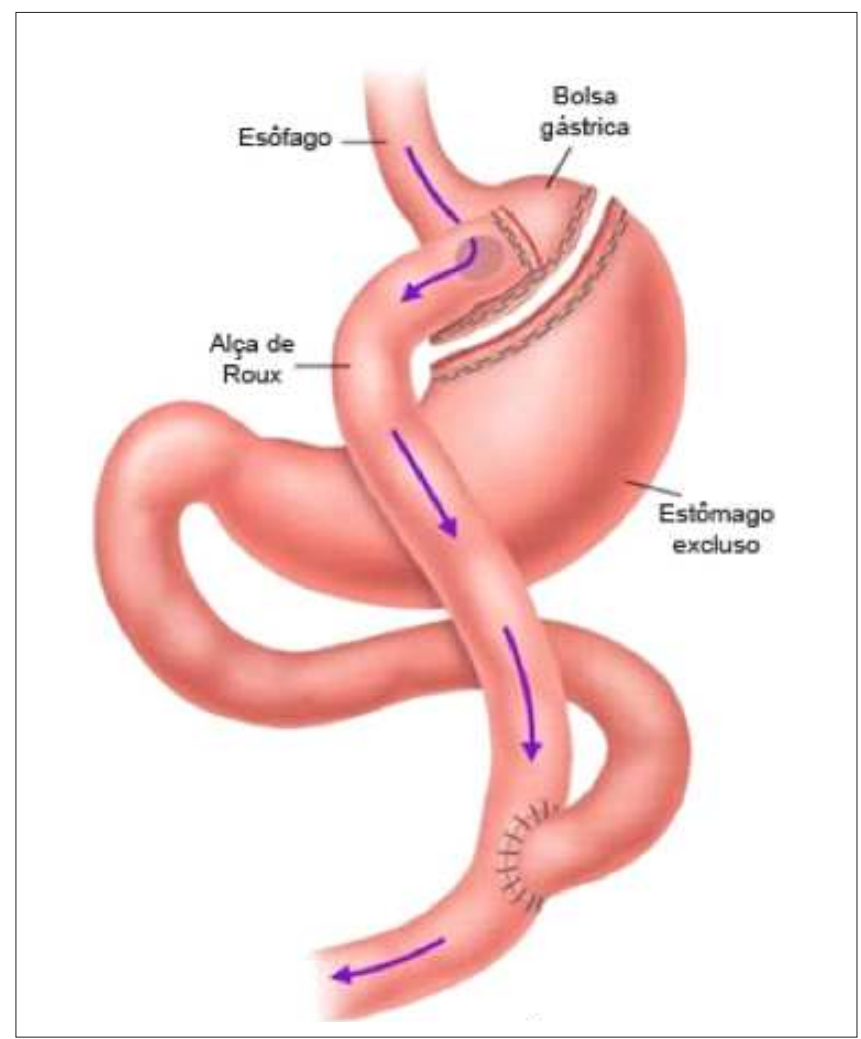

Figura 1 - Derivação gastrojejunal em Y-de-Roux.

\section{Variável insulina}

A insulina pré-operatória média foi de 29,6 6 IU/ $\mathrm{ml}$. No pós-operatório com 60 dias foi de 13,9 $\mu \mathrm{lU} / \mathrm{ml}$. A diferença pré e pós-operatória das mensurações foi de 15,7 $\mu \mathrm{lU} / \mathrm{ml}$, havendo diferença significativa entre elas $(p<0.001)$ (Figura 5).

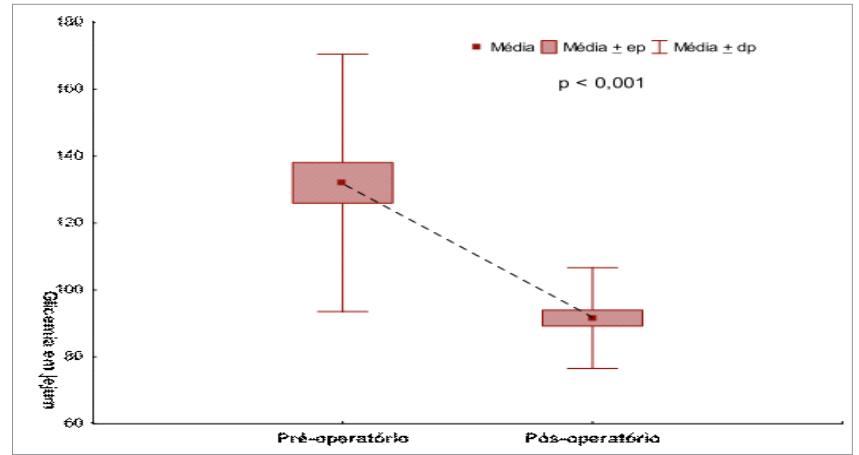

Figura 2 - Demonstração gráfica da glicemia em jejum segundo os períodos de avaliação.

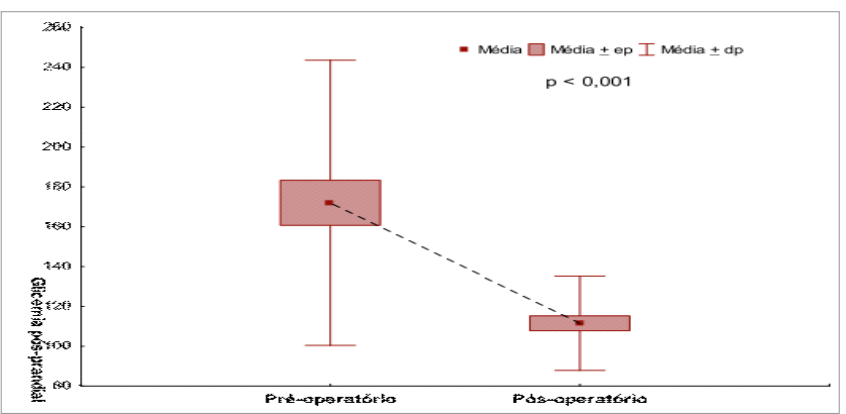

Figura 3 - Demonstração gráfica da glicemia pós-prandial segundo os períodos de avaliação.

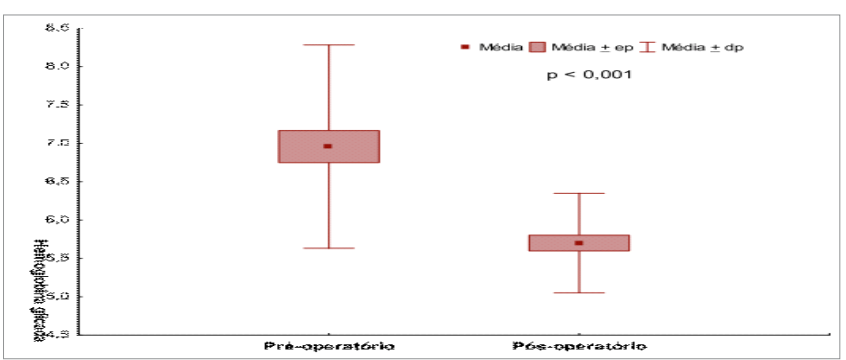

Figura 4 - Demonstração gráfica da hemoglobina glicada segundo os períodos de avaliação.

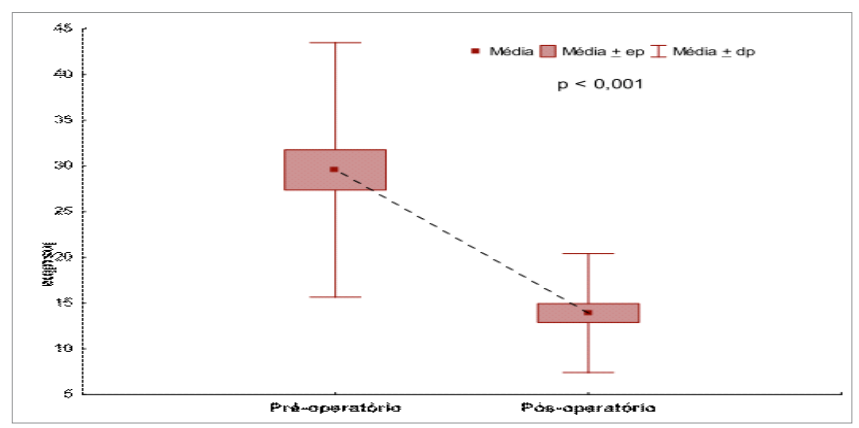

Figura 5 - Demonstração gráfica da insulina segundo os períodos de avaliação. 


\section{DISCUSSÃO}

A derivação gastrojejunal em Y-de-Roux é a operação para obesidade mórbida mais realizada no mundo, representando cerca de $65 \%$ de todos os procedimentos. Além do efeito mecânico restritivo e disabsortivo, ela promove diminuição estatisticamente significativa nos níveis pós-prandiais de grelina, importante hormônio orexígeno. Outros hormônios intestinais como o peptídeo YY e o GLP1 têm papel anorético e encontram-se aumentados após esse tipo de operação ${ }^{15}$. Ela atua inicialmente como operação restritiva, levando à saciedade precoce. A exclusao de parte do segmento estômago-duodeno-jejunal, leva à diminuição dos niìveis poìs-prandiais de grelina, diminuindo ainda mais o apetite. A presença mais precoce do alimento no iìleo terminal leva à maior produção de PYY e GLP-1, diminuindo a ingestão alimentar e otimizando o metabolismo glico-insuliìnico. Este é importante efeito antidiabetogênico dessa operação ${ }^{16,17}$.

A resolução do diabete acontece precocemente, antes mesmo que ocorra grande perda de peso. Tal fato pode ser explicado pelo efeito endócrino que esse procedimento produz, mesmo no período pós-operatório mais precoce $^{13,18}$

Corroborando com outros trabalhos, 15,19-24 os pacientes submetidos a derivação gastrojejunal em Y-deRoux sem anel tiveram redução significante dos parâmetros clínicos e laboratoriais 60 dias após o procedimento operatório apresentando normalização dos parâmetros laboratoriais analisados.

Quanto à idade e sexo, os resultados apresentados neste estudo confirmam os estudos de prevalência da obesidade e DM2 na população mundial ${ }^{15,25,26}$, onde $62,5 \%$ eram mulheres. A idade média foi de 41 anos variando de 24 a 62 anos.

A média pré-operatória do índice de massa corporal deste estudo foi de $39,5 \mathrm{Kg} / \mathrm{m}^{2} \mathrm{com}$ queda de 6,6 $\mathrm{Kg} / \mathrm{m}^{2}$ no IMC com 60 dias de pós-operatório e a perda de peso foi semelhante à pela literatura. ${ }^{15,23,26,27}$.

A glicemia média pré-operatória foi de $132 \mathrm{mg} /$ dl no pré-operatória diminuindo para $91,6 \mathrm{mg} / \mathrm{dl}$ com dois meses de pós-operatório. Semelhança aconteceu com a hemoglobina glicada a qual se encontra em 7,0\% no préoperatório e com 60 dias de pós-operatório já se encontrava em $5 \%$. Estes achados foram também referidos por outros autores $13,22,23,28-31$

Marina e Trence ${ }^{32}$, publicaram os resultados preliminares de um estudo onde sugeriu-se que a glicemia de jejum e a hemoglobina glicada não são critérios suficientes para determinar a remissão do DM2 após intervenção cirúrgica. As autoras sugerem que seja realizada monitorização glicêmica contínua e mensuração da glicemia pós-prandial para avaliação clara do perfil glicêmico, especificamente para observação dos efeitos da derivação gastrojejunal em Y-de-Roux nos pacientes com DM2.

Neste estudo, além do controle da glicemia de jejum e hemoglobina glicada, os valores da glicemia pósprandial também normalizaram. A glicemia pós-prandial pré-operatória média foi de $172 \mathrm{mg} / \mathrm{dl}$ diminuindo em média $60,5 \mathrm{mg} / \mathrm{dl}$ atingindo valores de 111,6 mg/dl com 60 dias de pós-operatório.

Em conclusão, houve significante diminuição de peso e no índice de massa corporal entre os períodos pré e pósoperatórios e diminuição também significante dos parâmetros laboratoriais de glicemia de jejum, glicemia pós-prandial, hemoglobina glicada, insulina entre os mesmos períodos.

\title{
A B S T R A C T
}

\begin{abstract}
Objectives: 1) To assess the changes in body weight and body mass index in grade 2 and 3 obese patients with type 2 diabetes mellitus, pre and postoperatively; 2) to assess the changes in pre and postoperative fasting blood glucose, postprandial glucose, glycated hemoglobin and insulin. Methods: A prospective study was undertaken with 40 selected patients with grade 2 and 3 obesity and type 2 diabetes mellitus, all with indication for surgical treatment of obesity. All patients underwent Roux-en-Y gastric bypass without ring. The clinical parameters weight and body mass index were analyzed, as well as the laboratory parameters fasting blood glucose, postprandial glucose, glycated hemoglobin, and insulin. The analyses were carried out in the preoperative period and at 60 days postoperatively. Statistical analysis was conducted with Student's $t$ and the Kolmogorov-Smirnov tests. Results: Mean preoperative weight was $107.3 \mathrm{~kg}$, declining to $89.5 \mathrm{~kg}$ postoperatively. Mean initial body mass index was $39.5 \mathrm{~kg} / \mathrm{m}^{2}$, and $32.9 \mathrm{~kg} / \mathrm{m}^{2}$ at 60 postoperative days. Preoperative fasting blood glucose was $132 \mathrm{mg} / \mathrm{dL}$, which was reduced to $40.4 \mathrm{mg} / \mathrm{dL}$, on average, postoperatively. Postprandial blood glucose was $172 \mathrm{mg} / \mathrm{dL}$ in the preoperative period and $111.6 \mathrm{mg} / \mathrm{dL}$ in the postoperative measurement. Initial glycated hemoglobin was 7\%, declining to $5.7 \%$ postoperatively. Preoperative and postoperative insulin levels were $29.6 \mu \mathrm{lU} / \mathrm{mL} \mathrm{and} 13.9 \mu \mathrm{lU} / \mathrm{mL}$, respectively. The level of significance was $p<0.001$ for all variables. Conclusion: There was a statistically significant reduction in body weight and body mass index between the pre and postoperative periods, as well as in fasting blood glucose, postprandial glucose, glycated hemoglobin.
\end{abstract}

Key words: Gastric bypass. Type 2 diabetes mellitus. Obesity. Bariatric surgery. 


\section{REFERENCIAS}

1. Fisberg M. Obesidade na infância e adolescência. In: XI Congresso Ciências do Desporto e Educação Física dos Países de Língua Portuguesa, 2006; São Paulo: Univerisidade de São Paulo; 2006 Set 06-09. p. 163-4. (Revista Brasileira de Educação Física e Esporte; vol. 20; supl. 5).

2. Mariath AB, Grillo LP, Silva RO, Schmitz P, Campos IC, Medina JRP, et al. Obesidade e fatores de risco para o desenvolvimento de doenças crônicas não transmissíveis entre usuários de unidade de alimentação e nutrição. Cad saúde pública. 2007;23(4):897-905.

3. Zhou L, Deng W, Zhou L, Fang P, He D, Zhang W, et al. Prevalence, incidence and risk factors of chronic heart failure in the type 2 diabetic population: systematic review. Curr Diabetes Rev. 2009;5(3):171-84

4. Laferrère B, Teixeira J, McGinty J, Tran H, Egger JR, Colarusso A, et al. Effect of weight loss by gastric bypass surgery versus hypocaloric diet on glucose and incretin levels in patients with type 2 diabetes. J Clin Endocrinol Metab. 2008;93(7):2479-85.

5. Mancini MC, Geloneze B, Salles JEN, Lima J, Carra MH, editores. Tratado de Obesidade. São Paulo: Guanabara Koogan; 2010.

6. Redmon JB, Reck KP, Raatz SK, Swanson JE, Kwong CA, Ji H, et al. Two-year outcome of a combination of weight loss therapies for type 2 diabetes. Diabetes Care. 2005:28(6):1311-5.

7. Pories WJ, Swanson MS, MacDonald KG, Long SB, Morris PG, Brown BM, et al. Who would have thought it? An operation proves to be the most effective therapy for adult-onset diabetes mellitus. Ann Surg. 1995;222(3):339-50; discussion 350-2.

8. Ferrannini $E$, Mingrone $G$. Impact of different bariatric surgical procedures on insulin action and beta-cell function in type 2 diabetes. Diabetes Care. 2009;32(3):514-20.

9. le Roux CW, Bloom SR. Why do patients lose weight after Rouxen-Y gastric bypass? J Clin Endocrinol Metab. 2005;90(1):591-2.

10. Geloneze B, Pareja JC. Cirurgia bariatrica cura a sindrome metabólica? Arq Bras Endocrinol Metab. 2006:50(2):400-7.

11. Polyzogopoulou EV, Kalfarentzos F, Vagenakis AG, Alexandrides TK. Restoration of euglycemia and normal acute insulin response to glucose in obese subjects with type 2 diabetes following bariatric surgery. Diabetes. 2003;52(5):1098-103.

12. Valverde I, Puente J, Martín-Duce A, Molina L, Lozano O, Sancho $V$, et al. Changes in glucagon-like peptide-1 (GLP-1) secretion after biliopancreatic diversion or vertical banded gastroplasty in obese subjects. Obes Surg. 2005;15(3):387-97.

13. Rubino F, Gagner M, Gentileschi P, Kini S, Fukuyama S, Feng J, et al. The early effect of the Roux-en-Y gastric bypass on hormones involved in body weight regulation and glucose metabolism. Ann Surg. 2004;240(2):236-42.

14. Capella JF, Capella RF. The weight reduction operation of choice: vertical banded gastroplasty or gastric bypass? Am J Surg. 1996;171(1):74-9.

15. Buchwald $H$, Avidor $Y$, Braunwald E, Jensen MD, Pories W, Fahrbach $\mathrm{K}$, et al. Bariatric surgery: a systematic review and meta-analysis. JAMA. 2004;292(14):1724-37.

16. Cummings $\mathrm{DE}$, Shannon $\mathrm{MH}$. Ghrelin and gastric bypass: is there a hormonal contribution to surgical weight loss? J Clin Endocrinol Metab. 2003;88(7):2999-3002.

17. Demaria EJ, Jamal MK. Surgical options for obesity. Gastroenterol Clin North Am. 2005;34(1):127-42.

18. Rubino F. Bariatric surgery: effects on glucose homeostasis. Curr Opin Clin Nutr Metab Care. 2006;9(4):497-507.

19. Schauer PR, Ikramuddin S, Gourash W, Ramanathan R, Luketich J. Outcomes after laparoscopic Roux-en-Y gastric bypass for morbid obesity. Ann Surg. 2000;232(4):515-29.
20. Sugerman HJ, Wolfe LG, Sica DA, Clore JN. Diabetes and hypertension in severe obesity and effects of gastric bypass-induced weight loss. Ann Surg. 2003;237(6):751-6; discussion 757-8.

21. Wittgrove AC, Clark GW. Laparoscopic gastric bypass, Roux-en-Y500 patients: technique and results, with 3-60 month follow-up. Obes Surg. 2000;10(3):233-9.

22. Schauer PR, Burguera B, Ikramuddin S, Cottam D, Gourash W, Hamad G, et al. Effect of laparoscopic Roux-en $Y$ gastric bypass on type 2 diabetes mellitus. Ann Surg. 2003;238(4):467-84; discussion 484-5.

23. Napoli TF, Guzzo MF, Hisano DK, Ribeiro PGFS, Guedes VJ, Ozawa $J C$, et al. Evaluation of weight loss and metabolic profile of obese patients with type 2 diabetes mellitus $(\mathrm{dm} 2)$ versus non- $\mathrm{dm} 2$ subjects, one and three years after bariatric surgery. $A B C D$ arq bras cir dig. 2010;23(2):100-4.

24. Cohen R, Pinheiro JS, Correa JL, Schiavon CA. Laparoscopic Rouxen-Y gastric bypass for BMI $<35 \mathrm{~kg} / \mathrm{m}(2)$ : a tailored approach. Surg Obes Relat Dis. 2006;2(3):401-4; discussion 404.

25. Garrido Júnior $A B$. Cirurgia em obesos mórbidos: experiência pessoal. Arq Bras Endocrinol Metab. 2000:44(1):106-10.

26. Milléo FQ, Malafaia O, Nassif PAN, Artoni RF, Santos MA. Estudo comparativo das técnicas cirúrgicas de Capella e Santoro tipo II para tratamento da obesidade, sobre o IMC e trigliceridemia periférica. Rev bras videocir. 2006:4(4):151-61.

27. Lin E, Davis SS, Srinivasan J, Sweeney JF, Ziegler TR, Phillips L, et al. Dual mechanism for type-2 diabetes resolution after Roux-en- $Y$ gastric bypass. Am Surg. 2009;75(6):498-502; discussion 502-3.

28. Gama MPR, Cardoso CR, Alves PP, Saito L, Suguiura CA, Piccolominni $A$, et al. Efeitos da cirurgia bariátrica em obesos mórbidos: redução da insulino resistência e melhora do perfil lipêmico. Endocrinol diabetes clin exp. 2002;2(2):13-20.

29. Morínigo R, Casamitjana R, Delgado S, Lacy A, Deulofeu R, Conget I, et al. Insulin resistance, inflammation, and the metabolic syndrome following Roux-en-Y gastric bypass surgery in severely obese subjects. Diabetes Care. 2007;30(7):1906-8.

30. Nassif PAN, Lopes AD, Lopes GL, Martins PR, Pedri LE, Varaschim $M$, et al. Alterações nos parâmetros pré e pós-operatórios de pacientes com síndrome metabólica, submetidos à bypass gastrointestinal em $Y$ de Roux. ABCD arq bras cir dig. 2009:22(3):165-70

31. Carvalho PS, Moreira CLCB, Barelli MC, Oliveira FH, Guzzo MF, Miguel GPS, et al. Cirurgia bariátrica cura síndrome metabólica? Arq Bras Endocrinol Metab. 2007;51(1):79-85

32. Marina AL, Trence DL. Is diabetes mellitus really cured by gastric bypass surgery? In: Meeting and Clinical Congress AACE, 2010; Boston: American Association of Clinical Endocrinologists; 2010 Abr 25. Abstract 210

Recebido em 20/09/2011

Aceito para publicação em 25/11/2011

Conflito de interesses: nenhum

Fonte de financiamento: nenhuma

\section{Como citar este artigo:}

Varaschim M, Nassif PAN, Moreira LB, Nascimento MM, Vieira GMN, Garcia RF, Sue KM, Cruz MA. Alterações dos parâmetros clínicos e laboratoriais em pacientes obesos com diabetes melito tipo 2 submetidos à derivação gastrojejunal em y de Roux sem anel. Rev Col Bras Cir. [periódico na Internet] 2012; 39(3). Disponível em URL: http:// www.scielo.br/rcbc

Endereço para correspondência:

Paulo Afonso Nunes Nassif

E-mail: paulonassif@terra.com.br 